\title{
Trimethoprim-Sulfamethoxazole-Induced Hypoglycemia as a Cause of Altered Mental Status in an Elderly Patient
}

\author{
Wayne A. Matbews, MS, PA-C, James E. Manint, DO, and Jobn Kleiss, RP
}

Drug-induced hypoglycemia is an important consideration in the differential diagnosis of altered mental status, especially in the elderly patient. Symptoms can be insidious, causing subtle mental status changes. In addition to the well known hypoglycemic effects associated with treatment of diabetes mellitus, several commonly prescribed medications, such as thiazides, sulfonylureas, and some antibiotics, can cause hypoglycemia. We describe a case of hypoglycemia in an elderly patient taking sulfonamide antibiotics. Previous reports in the medical literature have described this occurrence with sulfonamides, all in renally impaired patients or in those who were also taking sulfonylurea drugs. Although the incidence of drug-induced hypoglycemia is rare in the general population, its incidence is not uncommon in elderly and diabetic patients.

\section{Case Report}

A 91-year-old woman with a history of hypertension, transient ischemic attacks, and a recent urinary tract infection was brought to the emergency department in a decreased level of consciousness. Her history was obtained from the family, who accompanied her. They noted the patient could not be aroused that morning when they attempted to awaken her, and although she seemed alert the previous evening, some of her verbal statements were not making sense. Current medications included propoxyphene as needed, triamterene-hydrochlorothiazide once daily, and trimethoprim-sulfamethoxazole (TMP-SMX), double-strength, twice daily for the past 7 days. She had no known allergies.

Submitted, revised, 8 July 1999.

From the Kirby Hospital (WAM, JEM, JK) Monticello, IIl. Address reprint requests to Wayne A. Mathews, MS, PA-C, Kirby Hospital, 1111 N State St, Monticello, IL 61856.
The patient was obtunded on arrival at the hospital. She did not open her eyes to stimulation but did respond locally to painful stimuli. Her blood pressure was $200 / 91 \mathrm{~mm} \mathrm{Hg}$, heart rate 84 beats per minute in sinus rhythm, respiratory rate $16 /$ min, saturated oxygen on room air $91 \%$, and temperature $95.7^{\circ} \mathrm{F}$. During her neurologic examination, both pupils were $2 \mathrm{~mm}$ and equally reactive to light and accommodation. Deep tendon reflexes were $2+$ and symmetrical. She occasionally moved her left lower extremity, but no other voluntary or involuntary motor movement was noted. Her right foot had a positive, up-going, Babinski reflex.

She had no carotid bruits or thyromegaly. Her lung sounds were clear bilaterally on auscultation, and a grade $1 / 6$ systolic ejection murmur was heard at the right sternal border. Her abdomen was soft and not distended, and she had a normal pattern of bowel sounds in all quadrants. Her skin was cool but dry, with normal turgor. There was a small amount of dried blood on her lips and a small nonbleeding laceration on her tongue.

An electrocardiogram showed normal sinus rhythm and a heart rate of 82 beats per minute. There were no red blood cells or leukocytes in her urine, and it was negative for nitrates. Laboratory analysis of her blood showed the following values: creatinine $1.7 \mathrm{mg} . / \mathrm{dL}$, serum sodium $130 \mathrm{mEq} / \mathrm{L}$, potassium $4.1 \mathrm{mEq} / \mathrm{L}$, creatine kinase $301 \mathrm{U} / \mathrm{L}$, creatine kinase $\mathrm{MB}$ fraction $3.3 \%$, and plasma glucose $34 \mathrm{mg} / \mathrm{dL}$ before administration of intravenous dextrose. These results were obtained before noncontrast computed tomography (CT) of the brain was completed. The CT scan, which was performed after administration of intravenous dextrose, showed generalized cerebral atrophy with no focal bleeding, mass, or acute ischemic effect.

The patient was given one $50-\mathrm{mL}$ ampule of $50 \%$ dextrose solution intravenously. Within 5 minutes the patient opened her eyes and began to 
inquire where she was. Her speech was coherent; she was correctly oriented to person, place, and time; and she had no aphasia. There were no deficits found during motor or sensory examinations, although the Babinski reflex persisted. A finger-stick blood glucose reading 10 minutes later was $210 \mathrm{mg} / \mathrm{dL}$. She was admitted to the medical unit of the hospital for intravenous dextrose infusion and blood glucose monitoring, where she remained stable and continued to have regular findings on neurologic examinations.

\section{Discussion}

The differential diagnosis for delirium in this elderly patient would include sepsis, in this case a consideration because of the recent treatment for a urinary tract infection; cerebrovascular ischemia; seizure disorder; space-occupying brain mass; or endocrine disorders, such as diabetes mellitus or hypothyroidism; and drug-induced effects. The patient had no recent history of fever, falls, or headaches, and no known endocrine abnormality. The rapid response to intravenous dextrose indicated a primary hypoglycemic disorder secondary to the effects of recently prescribed sulfonamides.

A review of the literature for hypoglycemia caused by sulfonamide antibiotics in renally impaired patients found 10 cases of TMP-SMX- induced hypoglycemia. ${ }^{1}$ Two cases were reversible with dosage adjustment of the medication. Several risk factors for this unusual side effect include food deprivation, impaired renal function, and the concomitant use of sulfonylureas. A further consideration with this patient was the thiazide diuretic, which also contains a sulfa moiety. The sulfonamide antibiotics are related chemically to sulfonylureas, which are powerful hypoglycemic agents. Hypoglycemia has been reported to occur within 1.5 hours to 5 days of therapy with TMP-SMX. Although this patient was not diabetic, her estimated creatinine clearance for age and body weight was $18 \mathrm{~mL} / \mathrm{min}$. Product information for a commercial TMP-SMX preparation (Septra) recommends that patients with creatinine clearances of less than $30 \mathrm{~mL} / \mathrm{min}$ receive adjusted doses of the medication. ${ }^{2}$ It is postulated that the sulfamethoxazole portion binds to receptors of the pancreatic islet cells, causing the release of endogenous insulin. ${ }^{3}$

It is probable that this patient had a hypoglycemic seizure in the course of this episode, as evidenced by the tongue laceration and elevated creatine kinase level. Given the mental status changes the previous evening, her blood glucose was likely to have been steadily declining. The up-going Babinski reflex, which was indicative of a focal neurologic finding, in this case from a previous cerebrovascular infarction, was the reason for performing the noncontrast CT brain scan. The positive Babinski reflex served to confound the initial differential diagnosis, although no acute changes associated with cerebral infarction or cerebral bleeding were found on the CT scan. Because sulfonamide-induced hypoglycemia can be prolonged (approximately 12 hours), inpatient monitoring is indicated.

Several other medications, including thiazide diuretics, ethanol, aspirin, lithium, and angiotensinenzyme inhibitors, can also induce hypoglycemia. This patient was also taking a thiazide diuretic, which could have contributed with the sulfonamide to produce hypoglycemia. $\beta$-Blocker medications can also aggravate hypoglycemia by inhibiting the patient's awareness of hypoglycemic symptoms. A detailed medication history is essential for all patients coming to the emergency department with an altered mental status. All elderly patients taking sulfonamide antibiotics should have their renal function assessed and concomitant adjustment of medication dosage. Creatinine clearance can be estimated easily and accurately using the formula:

$(140$ - age)(weight in $\mathrm{kg})(0.85$ for women)/

\section{2 (serum creatinine in $\mathrm{mg} / \mathrm{dL}$ )}

This patient's hypoglycemia was the result of sulfonamide overdosage combined with the interactive side effect of hypoglycemia from the thiazide diuretic she was taking. Careful attention must be given both to adjusting dosages of medications to account for decreased renal function in the elderly and to being alert to potential interactive side effects from other medications.

\section{References}

1. Lee, AJ, Maddix, DS. Trimethoprim/sulfamethoxazole-induced hypoglycemia in a patient with acute renal failure. Ann Pharmacother 1997;31:727-32.

2. Septra product information insert. Research Triangle Park, NC: Burroughs Wellcome, 1998.

3. Hekimsoy Z, Biberoglu S, Comlekci A, Tarhan $\mathrm{O}$, Mermut C, Biberoglu K. Trimethoprim-sulfamethoxazole induced hypoglycemia in a malnourished patient with severe infection. Eur J Endocrinol 1997; 136:304-6. 